Rev. Elev. Méd. vét. Pays trop., 1973, 26 (1) : 93-116

\title{
Note sur la présence de Parabronema skrjabini Rassowska, 1924, chez des Ruminants sauvages d'Afrique
}

\author{
par P. M. TRONCY $(*)$, M. GRABER $\left({ }^{* *}\right)$ et J. THAL $\left(^{*}\right)$
}

\begin{abstract}
RESUME
Les auteurs étudient Parabronema skrjabini chez Hippotragus equinus d'Afrique Centrale: les mâles de ce matériel diffèrent du mâle type par la longueur inusitée du spicule gauche.
\end{abstract}

Décrit à l'origine en Asie Centrale chez le bétail domestique, ce parasite a, depuis, été rencontré à plusieurs reprises chez des animaux sauvages africains :

- Chez Okapia johnstoni (***): LEIPER (1935), Zoo de Londres [d'après ROUND (10)]; VUYLSTECKE (13), Zoo d'Anvers; Van den BERGHE et VUYLSTECKE (12), Zaïre; BAER (1), Zoos de Bâle et de Londres.

- Chez Capra ibex: EZZAT (5), Zoo de Giza.

- Chez Giraffa camelopardalis : EZZAT (5), Zoo de Giza; ORTLEPP (1961), Afrique du Sud [d'après ROUND (10)].

- Chez Syncerus caffer : DINNIK et collab. (4), Ouganda.

- Chez Hippotragus equinus: GRABER (6 et 7), Tchad.

Ce parasite n'a jamais été vu chez le bétail domestique d'Afrique; en Inde, au contraire,

(*) Laboratoire de Farcha, B.P. 433, Fort-Lamy, Tchad.

(**) Service de Parasitologie E.N.V., 2, quai Chauveau, 69337 Lyon, Cedex 1, France.

(***) Parfois associé à Okapinema okapi (Leiper, 1935) Ivashkin, 1960.
SARWAR (11), signale des infestations sévères chez les chèvres.

Il nous a paru intéressant de reprendre les études précédentes de l'espèce sur des points de détail, et de décrire les particularités propres à nos spécimens.

Matériel étudié : Le matériel que nous avons étudié provient du Tchad (Coll. BORREDON) et de République Centrafricaine (Coll. THAL).

a) Chez 2 Hippotragus equinus (DESMAREST, 1804) de R.C.A. Coordonnées des récoltes : $21-8-\mathrm{D}-\mathrm{d}$ et $21-9-\mathrm{D}-\mathrm{b}$.

(Environ 60 vers, mâles et femelles, en tout.)

b) Chez 1 Syncerus caffer (SPARRMANN, 1779) du Tchad. Coordonnées de récolte : 19 - 9 - A - b. (1 femelle unique, coparasite de Ashworthius lerouxi. Ce parasite semble tout à fait exceptionnel vu le grand nombre de buffles examinés.)

\section{Morphologie de l'extrémité céphalique}

Aucune des descriptions publiées à ce jour n'est semble-t-il correcte. SARWAR (11) a bien vu les 4 papilles latéro-médianes, mais il a placé les 4 papilles médio-médianes sur les pseudo-lèvres alors qu'elles se trouvent, diffi- 


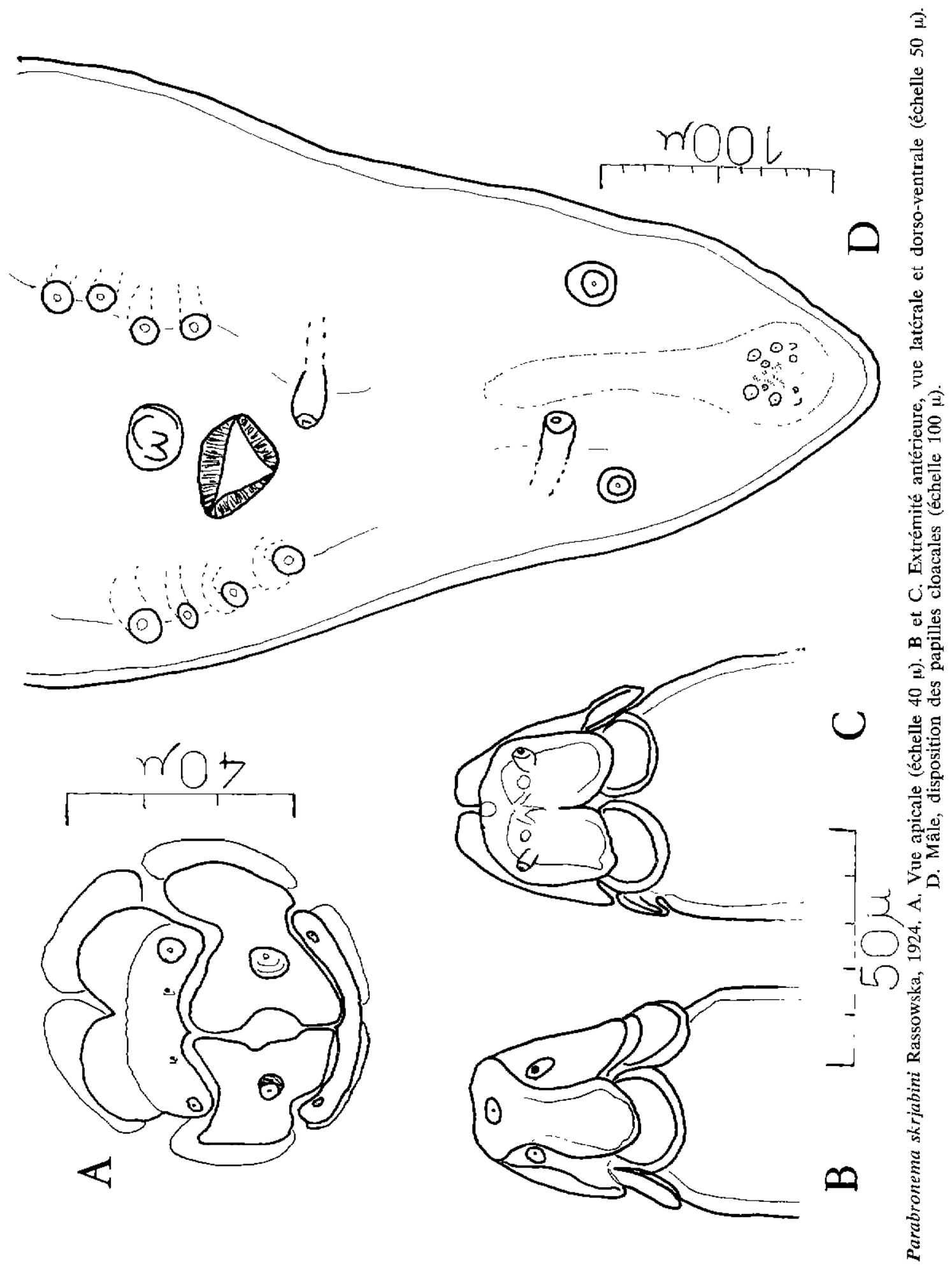


ciles à voir, sur les lèvres dorsale et ventrale (fig. $1 \mathrm{~A}, 1 \mathrm{~B}$ et $1 \mathrm{C}$ ).

\section{Disposition des papilles cloacales chez le mâle}

a) Contrairement aux descriptions précédentes, SARWAR (11) indique la présence de 5 paires de papilles précloacales et non de 4 .

Nous avons examiné de nombreux spécimens mâles, et avons constaté, dans 1 seul cas, la présence de 5 papilles précloacales à gauche et 4 à droite. Il semble donc y avoir une certaine variabilité, le chiffre de 4 paires de papilles étant, cependant, le chiffre normal (fig. 1 D).

b) Il existe bien un groupe terminal de 4 papilles, tel que SARWAR l'a dessiné (fig. 1 D); ce groupe de papilles est commun aux spirurides.

\section{Dimensions d'une femelle et d'un mâle}

a) Femelle. Longueur totale $23,8 \mathrm{~mm}$. Largeur maximale $200 \mu$. Longueur de l'œsophage
$2 \mathrm{~mm}$. Longueur de la capsule buccale $140 \mu$. Positions par rapport à l'apex de l'anneau nerveux : $250 \mu$; des deirides : $270 \mu$; du pore excréteur: $320 \mu$; de la vulve : $4,36 \mathrm{~mm}$. Longueur de la queue $160 \mu$.

b) Mâle. Longueur totale $12 \mathrm{~mm}$. Largeur maximale $170 \mu$. Longueur de l'œsophage $1,8 \mathrm{~mm}$. Longueur de la capsule buccale $130 \mu$. Positions par rapport à l'apex de l'anneau nerveux : $240 \mu$; des deirides : $240 \mu$; du pore excréteur : $300 \mu$. Longueur des spicules : gauche $1,38 \mathrm{~mm}$, droit $320 \mu$.

c) Discussion. Ces dimensions $\left({ }^{*}\right)$ s'accordent tout à fait avec celles données pour Parabronema skrjabini par RASSOWSKA (9), VUYLSTECKE (13), EZZAT (5) et SARWAR (11); sauf pour une mensuration: celle du spicule gauche, qui est de 600 à $710 \mu$ chez l'holotype. Sur 10 mâles examinés, nous avons relevé les dimensions suivantes pour les spicules :

\begin{tabular}{|c|cccccccccc|}
\hline $\begin{array}{c}\text { Spicule gauche } \\
\text { (en mm) }\end{array}$ & 1,41 & 1,4 & 1,38 & 1,28 & 1,37 & 1,27 & 1,45 & 1,2 & 1,27 & 1,35 \\
\hline $\begin{array}{c}\text { Spicule droit } \\
(\mathrm{en} \mu)\end{array}$ & 270 & 320 & 320 & 250 & 300 & 300 & 300 & 280 & 270 & 320 \\
\hline
\end{tabular}

CHABAUD (2) considère que la longueur des spicules a une valeur taxonomique dans la systématique du genre Parabronema; $P$. skrjabini fait partie des espèces dont le spicule gauche mesure moins de $1 \mathrm{~mm}$.

Cependant, le même auteur (3), trouvait chez $P$. africanum Baylis, 1921, une grande variabilité dans la longueur des spicules, le rapport spicule droit/spicule gauche pouvant varier de $1 / 4,5$ à $1 / 10$. Comme il n'y a pas de différence morphologique nette entre notre matériel et $P$. skrjabini, nous pensons que la longueur du spicule gauche n'a pas plus de valeur taxonomique dans le cas présent que dans le cas de $P$. africanum.

(*) Les dimensions de la femelle provenant de Syncerus caffer sont tout à fait comparables.
Il faut toutefois noter que $P$. skrjabini n'a jamais été trouvé chez des animaux domestiques d'Afrique, et qu'il semble particulièrement inféodé à l'Hippotrague. En effet, entre 1954 et 1968, GRABER (7) a examiné 6.622 zébus, 159 dromadaires, 5.682 moutons, 781 chèvres et 151 artiodactyles sauvages; il n'a rencontré P. skrjabini que chez Hippotragus equinus. Cette constatation est donc en contradiction avec les observations asiatiques, puisque dans ces pays le Parabronème est surtout un parasite d'animaux domestiques.

Pour toutes ces raisons, nous identifions notre matériel avec Parabronema skrjabini, seulement à titre provisoire, en attendant que ce parasite soit mieux connu en Afrique.

Les spécimens étudiés sont conservés au Muséum national d'Histoire naturelle sous les n $^{\circ} 41$ M.A. et 47 M.A. Bocal N 304. 


\section{SUMMARY}

Note on the presence of Parabronema skrjabini Rassowska, 1924, in african wild ruminants

The authors study $P$. skrjabini from $H$. equinus in Central Africa : males of this material are differents from the type species by the length of the left spicule.

\section{RESUMEN}

\section{Nota sobre la presencia de Parabronema skrjabini Rassowska, 1924, en los rumiantes salvajes de Africa}

Los autores estudian Parabronema skrjabini en Hippotragus equinus de Africa Central : los machos de dicho parásito difieren del macho tipo por la longitud inusitada de la espícula izquierda.

\section{BIBLIOGRAPHIE}

1. BAER (J. G.). Etude critique des helminthes parasites de l'Okapi. Acta trop., 1950, 7 (2) : 164-186.

2. CHABAUD (A.G.). Essai de classification des nématodes Habronematinae. Annls. Parasit. hum. comp., 1958, 33 (4) : 445-508.

3. CHABAUD (A. G.) et MOUCHET (J.). A propos d'un Spiruride, Parabronema africanum Baylis, 1921, présent dans le cour et le foie d'un éléphant; remarques sur la filiation des cycles évolutifs entre Spirurides et Filaires. Bull. Soc. Path. exot., 1956, 49 (2) : 467-472.

4. DINNIK (Y.), WALKER (J.B.), BARNETT (J. F.) et BROCKLESBY (D. W.). Some parasites obtained from game animals in western Uganda. Bull. epizoot. Dis. Afr., 1963, 13 (4) : 341-359.

5. EZZAT (M. abd E.). Helminth parasites of some ungulates from the Giza Zoological gardens, Egypt, with an appendix on some Nematodes from the African Rhinoceros. Techn. Sci. Service. Ministry Agric. Egypt., 1946, Bull, n 241, 104 p., 119 fig.

6. GRABER (M.), DOUTRE (M.), FINELLE (P.), KERAVEC (J.), DUCROZ (G.) et MOKOTAINGAR (P.). Les helminthes de quelques artiodactyles sauvages appartenant aux familles des bovidés et des suidés. Ces mammıfères, en République du Tchad et en R.C.A. sont-ils des réservoirs de parasites pour les animaux domes- tiques vivant à leur contact? Rev. Elev. Méd. vét. Pays trop., 1964, 17 (3): 377-419.

7. GRABER (M.). Helminthes parasites de certains animaux domestiques et sauvages du Tchad. Bull, epizoot. Dis. Afr., 1969, 17 (4) : 403-428.

8. IVASHKIN (V.M.). Nématodes de la sousfamille Parabronematinae Skrjabin, 1941, d'après leur morphologie et leur systématique (en russe). Trudy gel'mint. Lab. Akad. Nauk SSR, 1960, 10: 94-108.

9. RASSOWSKA (P. I.). Nématodes du gros bétail à corne du Turkestan (en russe). Trudy. gosudarstv. Inst. Eksper. Vet., 1924, 2 : 129-136.

10. ROUND (M.C.). Check list of the Helminth Parasites of African Mammals. Commonwealth Bureau of Helminthology, St Albans, Technical Communication, 1968, 38, 252 p.

11. SARWAR (M. M.). On the tranference of Parabronema skrjabini Rassowska, 1924, to Squamanema Thiel, 1925, and its Occurence in Sheep and Goats in Indo-Pakistan. J. Helminth., 1954, $28(3 / 4): 143-150$.

12. Van den BERGHE (L.) et VUYLSTECKE (C.). Contribution à l'étude des parasites de l'Okapi (deuxième partie). Revue Zool. Bot. afr., 1937, 29 (4) : 361-369.

13. VUYLSTECKE (C.). Nématodes parasites de l'Okapi. Rev. Zool. Bot. afr., 1935, 27 (3/4) : 313318. 